\title{
Erratum to "Cryptanalysis of the birational permutation signature scheme over a non-commutative ring" [JSIAM Letters, 2 (2010), 85-88]
}

Naoki Ogura ${ }^{1}$ and Shigenori Uchiyama ${ }^{1}$

\footnotetext{
${ }^{1}$ Department of Mathematics and Information Sciences, Tokyo Metropolitan University, Tokyo 192-0397, Japan

Received September 13, 2010, Accepted September 14, 2010
}

The name of the second author was wrongly printed as "Uchiyama Shigenori", but it should be read as "Shigenori Uchiyama" as shown here. 